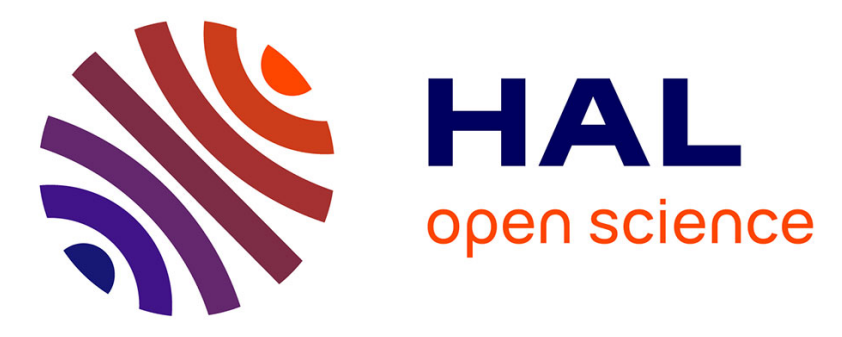

\title{
A Novel Constellation Shaping Technique for Bit-Interleaved Coded Modulation
}

Bouazza Boubakar, Legoff Stéphane Y., Garadi Ahmed, Clency Perrine, Rodolphe Vauzelle

\section{- To cite this version:}

Bouazza Boubakar, Legoff Stéphane Y., Garadi Ahmed, Clency Perrine, Rodolphe Vauzelle. A Novel Constellation Shaping Technique for Bit-Interleaved Coded Modulation. Wireless Personal Communications, 2013, 71 (2), 10 p. 10.1007/s11277-013-1303-9 . hal-00909755

\section{HAL Id: hal-00909755 https://hal.science/hal-00909755}

Submitted on 29 Nov 2013

HAL is a multi-disciplinary open access archive for the deposit and dissemination of scientific research documents, whether they are published or not. The documents may come from teaching and research institutions in France or abroad, or from public or private research centers.
L'archive ouverte pluridisciplinaire HAL, est destinée au dépôt et à la diffusion de documents scientifiques de niveau recherche, publiés ou non, émanant des établissements d'enseignement et de recherche français ou étrangers, des laboratoires publics ou privés. 


\title{
A Novel Constellation Shaping Technique for Bit-Interleaved Coded Modulation
}

\author{
Boubakar S. Bouazza · Stéphane Y. LeGoff • \\ Ahmed Garadi · Clency Perrine · Rodolphe Vauzelle
}

(C) Springer Science+Business Media New York 2013

\begin{abstract}
We present a new and simple method which consists to apply constellation shaping to bit-interleaved turbo-coded modulation (BICTM) over additive white Gaussian noise channels. By assuming the example of a 3-bit/dim 16-PAM BITCM, it is shown that this technique can provide shaping gain of $0.79 \mathrm{~dB}$.
\end{abstract}

Keywords Constellation Shaping · Bit-interleaved coded modulation (BICM)

\section{Introduction}

For the Gaussian channel, constellation shaping techniques refer to the selection of the signal shape where the average energy is reduced and the constellation shape is Gaussian in distribution [1,2]. Thus, the system gain is obtained by the sum of both coding gain and shaping gain. For bandwidth-efficient communications over additive white Gaussian noise (AWGN) channels, power and bandwidth are the two main constraints. To overcome the problem of large needed powers, it is interesting to investigate the combination of constellation shaping and turbo coding [3]. Some techniques have recently been proposed to apply constellation shaping principles to bit-interleaved coded modulation (BICM) schemes using turbo coding, referred hereafter to as bit-interleaved turbo-coded modulation (BITCM) schemes [4-7].

In this paper, we present another method for combining shaping and BITCM. Our technique is based on a shaping technique in which the basic constellation ispartitioned into

B. S. Bouazza $(\varangle) \cdot$ A. Garadi

Laboratory Technology of Communications, University of Tahar Moulay Saida, Saida, Algeria e-mail: bsbouazza@yahoo.fr

S. Y. LeGoff

School of Electrical, Electronic and Computer Engineering, Newcastle University,

Newcastle upon Tyne, UK

C. Perrine $\cdot$ R. Vauzelle

Department of Signal Image and Communications, XLIM Institute,

University of Poitiers, CNRS JUR 7252, Poitiers, France 
several equal-sized sub-constellation of increasing average energy [8]. It consists of counting the number of zeros (0) at a certain level of the output of the turbo coder. The number of zeros (0) should always exceed the number of ones (1) in order to specify the sequence of sub-constellation so that low-energy signals are transmitted more frequently than high-energy signals. The partitioning method preserves the Gray mapping provided that only one level of partition is considered, i.e. the basic constellation is divided into two sub-constellations. This relative compatibility between shaping and Gray mapping constitutes a crucial point since it is well known that BITCM schemes perform optimally when Gray mapping is used to label constellation signal points [9]. Throughout this work, we assume a Gaussian channel, and only consider the case of $2^{m}$-ary one-dimensional (1-D) constellation, hereafter referred to as $2^{m}$-PAM constellation which is equivalent to an $2^{2 . m}$-QAM.

The organization of this paper is as follows: In Sect. 2, the structures of our BICM transmitter and receiver are presented. Computer simulation results are shown in Sect. 3. Finally, conclusions are drawn in Sect. 4.

\section{System Description}

In this section, we introduce the structure of the proposed system that combines constellation shaping and BICM. Hereafter, a notation in the form $X=\left(x_{n}\right)_{N}$ shall be used to denote a vector $X$ composed of $N$ scalar quantities $x_{n}, n \in\{1,2, \ldots, N\}$.

\subsection{The Proposed BICM Transmitter with Constellation Shaping}

Consider a bandwidth-efficient communication system using $2^{m}$-ary pulse-amplitude modulation (PAM) and operating over an additive white Gaussian noise (AWGN) channel. Gray mapping is employed to label constellation signal points, as is generally the case in BICM. The block diagram of the proposed transmitter is given in Fig. 1.

Consider the transmission of a vector of $N$ information bits. This sequence is first encoded using a rate- $R_{c}$ error-correcting encoder. The vector $\mathcal{C}$ thus obtained is converted into $\mathrm{m}$ parallel binary vectors which are then interleaved in a random fashion $\pi_{i}, i \epsilon\{1, \ldots, m\}$ to produce $m$ vectors $\mathcal{C}_{i}, i \epsilon\{1, \ldots, m\}$. The serial-to-parallel (SP) conversion is performed so that the length of the first vector $\mathcal{C}_{1}$ is shorter than that of the other $(m-1)$ vectors $\mathcal{C}_{i}, i \epsilon\{2, \ldots, m\}$.

If $L$ designates the length of a vector $\mathcal{C}_{i}, i \epsilon\{1, \ldots, m\}$, the length of $\mathcal{C}_{1}$ is then given by $L_{1}=L \cdot \frac{(Q-1)}{Q}<L$ where $Q$ is an integer whose practical significance is explained in the

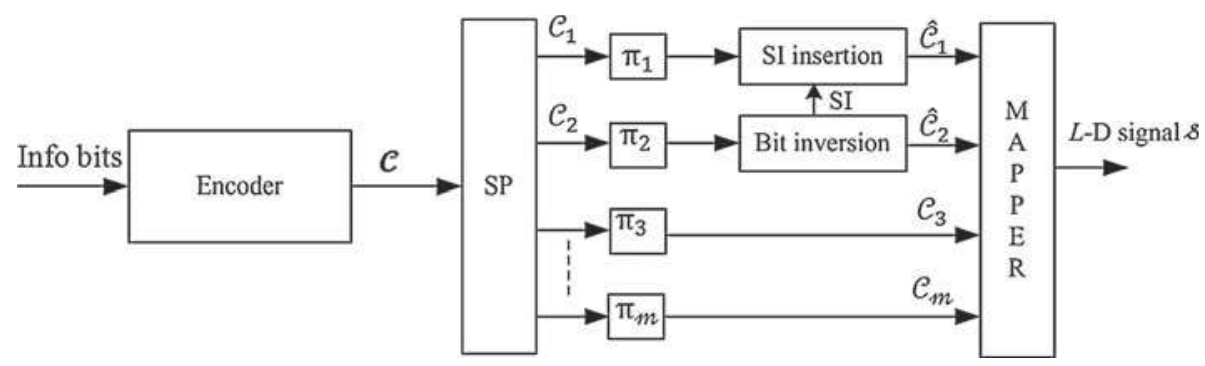

Fig. 1 Block diagram of the proposed BICM transmitter 


\section{Author's personal copy}

A Novel Constellation Shaping Technique

Table 1 Probability $P_{0}$ of zero in the sub-vectors generated by the inversion block as a function of the length $Q$ of these sub-vectors for $Q$ ranging from 2 to 11

\begin{tabular}{llllll}
\hline$Q$ & 2,3 & 4,5 & 6,7 & 8,9 & 10,11 \\
$P_{0}$ & 0.7500 & 0.6875 & 0.6562 & 0.6367 & 0.6230 \\
The saving energy & 2.06 & 1.43 & 1.16 & 0.99 & 0.89 \\
\hline
\end{tabular}

next paragraph. Let us focus now on the second binary vector $\mathcal{C}_{2}$. This $L$-bit vector is first divided into a sequence of $Q$-bit sub-vectors.

Let $\mathcal{C}_{2, Q}=\left(c_{q}\right)_{Q}$ denote a particular sub-vector composed of $Q$ bits $c_{q}, q \in\{1, \ldots, Q\}$. Each sub-vector is then individually processed by a bit inversion block as follows: The number of zeros and ones in $\mathcal{C}_{2, Q}$ is first counted. If the block detects that there are more ones than zeros in $\mathcal{C}_{2, Q}$, all bits $c_{q}, q \in\{1, \ldots, Q\}$ are inverted. If this is not the case, this inversion does not take place. As a summary, for every sub-vector $\mathcal{C}_{2, Q}$, the inversion block generates a corresponding $Q$-bit sub-vector $\widehat{\mathcal{C}}_{2, Q}=\left(\widehat{c}_{q}\right)_{Q}$ equal to either $\mathcal{C}_{2, Q}=\left(c_{q}\right)_{Q}$ or $\overline{\mathcal{C}}_{2, Q}=\left(\bar{c}_{q}\right)_{Q}$ depending on the number of zeros in $\mathcal{C}_{2, Q}$. The result is that the successive sub-vectors $\widehat{\mathcal{C}_{2}, Q}$ available at the inversion block output always contain more zeros than ones.

It is important to mention that the probability $P_{0}$ of a zero in the $L$-bit vector $\widehat{\mathcal{C}_{2}}$, which is composed of the successive sub-vectors $\widehat{\mathcal{C}}_{2}, Q$, depends on the value of the parameter $Q$ i.e., the value of $P_{0}$ decreases as the value of $Q$ is increased. A computer program was whiten so as to calculate $P_{0}$ as the function of $Q$, Table 1 shows the values of $P_{0}$ obtained when $Q$ ranges from 2 to 11 , which is equal to

$$
P_{0}=\frac{1}{Q} \sum_{q=1}^{Q} \operatorname{Pr} .\left[\widehat{c}_{q}=0\right]
$$

The bit inversion block also generates, for each sub-vector $\mathcal{C}_{2, Q}$, an additional bit, referred to as side information (SI) bit, to indicate whether or not an inversion of $\mathcal{C}_{2, Q}$ has been performed. This SI bit must also be transmitted as the receiver requires an estimate of its value in order to operate properly. For each $L$-bit vector $\mathcal{C}_{2}$, the total number of SI bits is equal to $L / Q$ We transmit these SI bits by inserting them inside the first vector $\mathcal{C}_{1}$. Since the original length of $\mathcal{C}_{1}$ is given by $L_{1}=L \cdot \frac{Q-1}{Q}<L$, the insertion of the SI bits in $\mathcal{C}_{1}$ produces another vector $\widehat{\mathcal{C}}_{1}$ whose length is now equal to $L_{1}=L \cdot \frac{Q-1}{Q}+\frac{L}{Q}=L$ bits. The reason why we choose to insert the SI bits inside $\mathcal{C}_{1}$ rather than in any other vector $\mathcal{C}_{i}, i \epsilon\{2, \ldots, m\}$ will be explained later. Note that, in order to operate properly, the receiver requires very reliable estimates of these SI bits, since a whole $Q$-bit subvector $\mathcal{C}_{2, Q}$ is, in effect, lost every time the corresponding SI bit is erroneously detected. It is worthwhile mentioning that the binary vectors $\mathcal{C}_{i}, i \in\{3, \ldots, m\}$, are not processed at all, unlike $\mathcal{C}_{1}$ and $\mathcal{C}_{2}$. Finally, a vector $\left(c_{j, 1}, c_{j, 2}, \ldots, c_{j, m}\right)$, where $c_{j, i}$ denotes the $\mathrm{j}$-th bit of either $\widehat{\mathcal{C}}_{i}, i \epsilon\{1,2\}$, or $\mathcal{C}_{i}, i \epsilon\{3, \ldots, m\}$ is mapped onto a signal point of a $2^{m}$-PAM constellation according to Gray labelling. We can easily show that the transmission of $N=R_{c} \cdot\left((m-1)+\frac{Q-1}{Q}\right) . L$ information bits is performed by emitting $L$ successive 1-D signal points $s_{j}, j \in\{1, \ldots, L\}$ i.e. an $L$-dimensional $(L$-D) signal point denoted $s$. Hence, the data rate $R$ obtained with the proposed system is

$$
R=R_{c} \cdot\left(\frac{Q \cdot m-1}{Q}\right) \text { bits } / \mathrm{dim}
$$

which is less than the rate $R=R_{c}$ bits/dim obtained with an equivalent BICM scheme designed using the traditional method. This loss in data rate, which is due to the presence of 
B. S. Bouazza et al.

\begin{tabular}{|c|c|c|c|c|c|c|c|c|c|c|c|c|c|c|c|c|}
\hline$s_{j}$ & -15 & -13 & -11 & -9 & -7 & -5 & -3 & -1 & I & 3 & $\begin{array}{l}5 \\
1\end{array}$ & 7 & 9 & 11 & 13 & 15 \\
\hline & $\mid$ & 1 & 1 & 1 & 1 & 1 & 1 & 1 & 1 & 1 & | & T & 1 & $T$ & 1 & 1 \\
\hline$c_{j, 1}$ & 0 & 0 & 0 & 0 & 0 & 0 & 0 & 0 & 1 & 1 & 1 & 1 & 1 & 1 & 1 & 1 \\
\hline$c_{j, 2}$ & 1 & 1 & 1 & 1 & 0 & 0 & 0 & 0 & 0 & 0 & 0 & 0 & 1 & 1 & 1 & 1 \\
\hline$c_{j, 3}$ & 1 & 1 & 0 & 0 & 0 & 0 & 1 & 1 & 1 & 1 & 0 & 0 & 0 & 0 & 1 & 1 \\
\hline$c_{j, 4}$ & 1 & 0 & 0 & 1 & 1 & 0 & 0 & 1 & 1 & 0 & 0 & 1 & 1 & 0 & 0 & 1 \\
\hline
\end{tabular}

Fig. 2 Gray labeling for 16-PAM constellation $(m=4)$

the SI bits inside the transmitted sequence, is compensated by increasing the coding rate of the error correcting code by a factor equal to $\frac{Q . m}{Q . m-1}$.

To understand how the proposed system can achieve better error performance than a traditional BICM scheme,we need to have a close look at some characteristics of the Gray mapping for PAM constellations. As an example, Fig. 2 shows the Gray labelling for 16-PAM constellation, corresponding to the case $m=4$. Figure 2 clearly shows that, in Gray mapping, some bits are inherently better protected than others from the detrimental effect of Gaussian noise (see, e.g., [10] for more details). Actually, we can see that the protection offered to bits $c_{j, i}, i \in\{1, \ldots, m\}$, decreases as the value of $i$ increases, thus implying that there are $m$ different layers of protection. The first layer, corresponding to $i=1$, should therefore be used to carry bits that must be transmitted with the highest possible reliability, such as the SI bits generated by the inversion block in the proposed BICM scheme. As the sequence transmitted over the first layer of protection is the vector $\widehat{\mathcal{C}}_{1}$, the best strategy is definitely to embed the SI bits in $\widehat{\mathcal{C}}_{1}$.

From Fig. 2, we also notice that the second layer, corresponding to $i=2$, presents the following characteristic: one of the $2^{m-1}$ (=8 in Fig. 2) signal points of lowest energies in the constellation is selected every time we have $c_{j, 2}=0$. The fact that $\operatorname{Pr}\left[c_{j, 2}=0\right]>\operatorname{Pr}\left[c_{j, 2}=\right.$ 1], or equivalently $P_{0}>0.5$, due to the use of the inversion block, means that the $2^{m-1}$ lowest-energy signals are transmitted more frequently than the $2^{m-1}$ highest-energy signals, thus achieving a constellation shaping effect similar to that described in $[6,8]$.

This results in some saving in the average energy per transmitted signal when compared to a classical BICM transmitter (for which $P_{0}=0.5$ ). In fact, the average energy $\gamma$ per transmitted signal point $s_{j}, j \in\{1, \ldots, L\}$ is given by

$$
\gamma=\gamma_{0} \cdot P_{0}+\gamma_{1} \cdot\left(1-P_{0}\right)
$$

where $\gamma_{0}$ and $\gamma_{1}$ are the average energies of the $2^{m-1}$ lowest-energy signals and $2^{m-1}$ highestenergy signals, respectively. As $\gamma_{0}<\gamma_{1}$ and $P_{0}>0.5$, we can easily show that $\gamma_{0}<\dot{\gamma}$, where $\dot{\gamma}=\frac{\gamma_{0}+\gamma_{1}}{2}$ designates the average energy per transmitted signal point in an equivalent BICM scheme without constellation shaping, i.e. for which all signal points in the PAM constellation are equiprobable $\left(P_{0}=0.5\right)$. In other words, the use of the bit inversion block reduces the average energy per transmitted signal by a factor equal to

$$
\frac{\dot{\gamma}}{\gamma}=\frac{1}{2} \cdot \frac{\gamma_{0}+\gamma_{1}}{\gamma_{0} \cdot P_{0}+\gamma_{1} \cdot\left(1-P_{0}\right)}
$$

For instance, if we assume $Q=6$ and the use of 16-PAM ( $m=4$ ) we can show that $\gamma_{0}=21, \gamma_{1}=149, P_{0} \approx 0.6562$ (see Table 1 ). In this case, the energy saving achieved using the proposed technique is equal to $10 . \log _{10}\left[\frac{\hat{\gamma}}{\gamma}\right] \approx 1.16 \mathrm{~dB}$.

Unfortunately, this energy saving does not directly translate into a shaping gain at the receiver output. In fact, the shaping gain is smaller than the energy saving because of two reasons: 


\section{Author's personal copy}

A Novel Constellation Shaping Technique

- The coding rate $R_{c}$ needs to be increased to compensate for the rate loss due to the side information, thus reducing the error-correction capabilities of the error-correcting code;

- The error rate on the transmitted SI bits is not equal to zero.

\subsection{The Proposed BICM Receiver}

The block diagram of the proposed BICM receiver is depicted in Fig. 3. The received $L$-D signal $r$ is a vector of $L$ channel samples $r_{j}, j \in\{1, \ldots, L\}$ expressed as $r_{j}=s_{j}+w_{j}$, where $w_{j}$ is a Gaussian noise sample with zero mean and variance $\sigma^{2}$. In the de-mapping block, the logarithm of likelihood ratio (LLR) $L\left(c_{j, i}\right)$ associated with each bit $c_{j, i}, i \epsilon\{1, \ldots, m\}$ is computed from sample $r_{j}$ using well-known expressions, such as the simplified equations proposed in [11].

The fact that $\operatorname{Pr}\left[c_{j, 2}=0\right] \neq \operatorname{Pr}\left[c_{j, 1}=1\right]$ is due to the use of the inversion block at the transmitter side must be taken into account when evaluating the LLRs $L\left(c_{j, 2}\right), j \in\{1, \ldots, L\}$. We can demonstrate that it can be done by simply adding, to the standard expressions obtained for $\operatorname{Pr}\left[c_{j, 1}=0\right]=\operatorname{Pr}\left[c_{j, 1}=1\right]$, a constant computed as

$$
\ln \left[\frac{\operatorname{Pr}\left[c_{j, 2}=1\right]}{\operatorname{Pr}\left[c_{j, 2}=0\right]}\right]=\ln \left[\frac{1-P_{0}}{P_{0}}\right]
$$

Note that this term only depends on the value of the parameter $Q$ (since $P_{0}$ only depends on $Q$ ). As an example, if we use the simplified LLR equations introduced in [11], the LLRs $c_{j, i}, j \in\{1, \ldots, L\}$, are given by

$$
L\left(c_{j, 2}\right)=\left|r_{j}\right|-2^{m-1}+\ln \left[\frac{1-P_{0}}{P_{0}}\right]
$$

where the operator $\left|r_{j}\right|$ denotes absolute value of $r_{j}$.

The LLRs of the SI bits (denoted as $L(\mathrm{SI})$ in Fig. 3) are extracted from the vector $L\left(\widehat{\mathcal{C}_{1}}\right)$ composed of the LLRs $L\left(c_{j, 1}\right), j \in\{1, \ldots, L\}$, and then fed into the soft inversion block. The task of this block is to process the LLRs $L\left(c_{j, 2}\right), j \epsilon\{1, \ldots, L\}$ initially computed by the de-mapper,by taking into account the LLRs of the SI bits. To explain how the soft inversion block operates, let us focus on a particular bit $\widehat{c}_{q}$ of a transmitted sub-vector $\widehat{c}_{2, Q}=\left(\widehat{c}_{q}\right)_{Q}$. We have previously seen that we have either $\widehat{c}_{q}=c_{q}$ or $\widehat{c}_{q}=\bar{c}_{q}$.

It can therefore be shown that the LLR $L\left(c_{q}\right)$ is either given by $L\left(c_{q}\right)=L\left(\widehat{c}_{q}\right)$ or $L\left(c_{q}\right)=-L\left(\widehat{c}_{q}\right)$ where $L\left(\widehat{c}_{q}\right)$ is the estimate of $\widehat{c}_{q}$ produced by the de-mapper [12]. The receiver uses the estimate of the corresponding SI bit, hereafter denoted as $c_{\text {SI }}$, to evaluate $L\left(c_{q}\right)$ using the generic expression

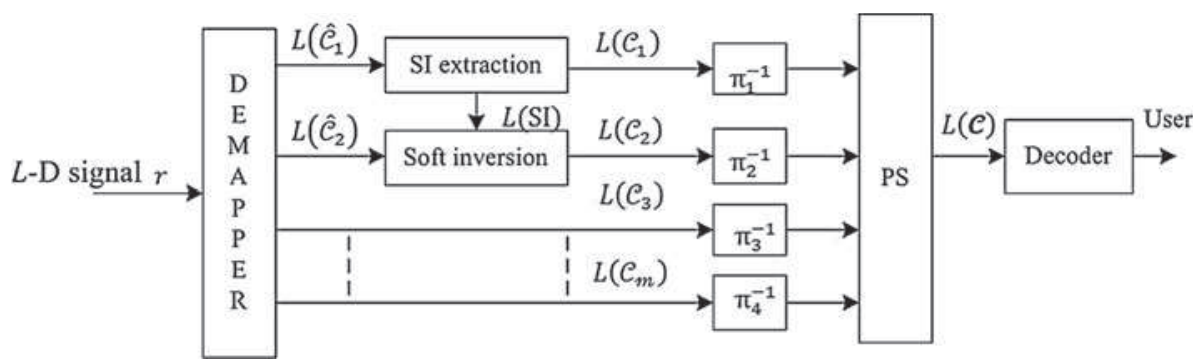

Fig. 3 Block diagram of the proposed BICM receiver 


$$
L\left(c_{q}\right)=L\left(\widehat{c}_{q}\right) \cdot \operatorname{Pr}\left[c_{\mathrm{SI}}=0\right]-L\left(\widehat{c}_{q}\right) \cdot \operatorname{Pr}\left[c_{\mathrm{SI}}=1\right]
$$

obtained by assuming that $\widehat{c}_{q}=c_{q}$ corresponds to $c_{\mathrm{SI}}=0$ and $\widehat{c}_{q}=\bar{c}_{q}$ is associated with $c_{\mathrm{SI}}=1$. In addition, we can show that [12]

$$
\operatorname{Pr}\left[c_{\mathrm{SI}}=k\right]=\frac{e^{k \cdot L\left(c_{\mathrm{SI}}\right)}}{1+e^{k \cdot L\left(c_{\mathrm{SI}}\right)}} \quad \text { for } k \epsilon\{0,1\}
$$

By combining (6) and (7), we obtain

$$
L\left(c_{q}\right)=L\left(\widehat{c}_{q}\right) \cdot \frac{1-e^{k \cdot L\left(c_{\mathrm{SI}}\right)}}{1+e^{k \cdot L\left(c_{\mathrm{SI}}\right)}}
$$

The task of the soft inversion block is thus to compute the LLR $L\left(c_{q}\right)$ using both estimates $L\left(\widehat{c}_{q}\right)$ and $L\left(c_{S I}\right)$ based on (9). Note that, if the simplified LLR equations derived in [11] are employed, (9) becomes:

$$
L\left(c_{q}\right)=L\left(\widehat{c}_{q}\right) \cdot\left[\frac{1-e^{r_{\mathrm{SI}}}}{1+e^{r_{\mathrm{SI}}}}\right]
$$

where $r_{\mathrm{SI}}$ is the channel sample carrying the SI bit. Note that (10) is even simpler to implement than (9). The parallel sequences $L\left(\mathcal{C}_{i}\right)$ composed of the LLRs $L\left(c_{j, i}\right), i \in\{1, \ldots, m\}$ and $j \epsilon\{1, \ldots, m\}$ are de-interleaved and then converted into a serial stream $L(\mathcal{C})$ of LLRs. The latter is finally decoded using a soft-decision decoder.

\section{Design of a 3-bit/dim 16-PAM BITCM Scheme}

In this section, we consider the example of 3-bit/dim 16-PAM BITCM for which we provide theoretical analysis in addition to computer simulation results.

\subsection{Theoretical Shaping Gain}

The achievable shaping gains can be determined by evaluating the gains in terms of mutual information obtained with the proposed shaping algorithm. Let $s \in S$ and $r$ denote respectively the transmitted signal and the corresponding received signal.

Information theory tells us that the mutual information $I$ of the discrete-input Gaussian channel, expressed in bit/dim, is given by [6]

$$
I=\sum_{s \in S} \int_{-\infty}^{+\infty} \operatorname{Pr}(s) p(r \mid s) \log _{2}\left[\frac{p(r \mid s)}{\sum_{s \in S} \operatorname{Pr}(s) p(r \mid s)}\right] d r
$$

where $p(r \mid s)$ denotes the transition probability density function of the gaussian channel. It can easily be shown that, for the system described in this paper, (11) is equivalent to:

$$
I=E_{S, r}\left[\log _{2}\left(\frac{2^{m-1} \exp \left(-N_{0}^{-1}(r-s)^{2}\right)}{\sum_{x \in S} P(x) \exp \left(-N_{0}^{-1}(r-x)^{2}\right)}\right)\right]
$$

where $E_{s, r}$ denotes expectation respect to $s$ and $r$, and the term $P(\mathrm{x})$ is given by:

$$
P(x)= \begin{cases}\left(1-P_{0}\right) & \text { for } x \in S_{1} \\ P_{0} \longrightarrow & \text { for } x \in S_{0}\end{cases}
$$




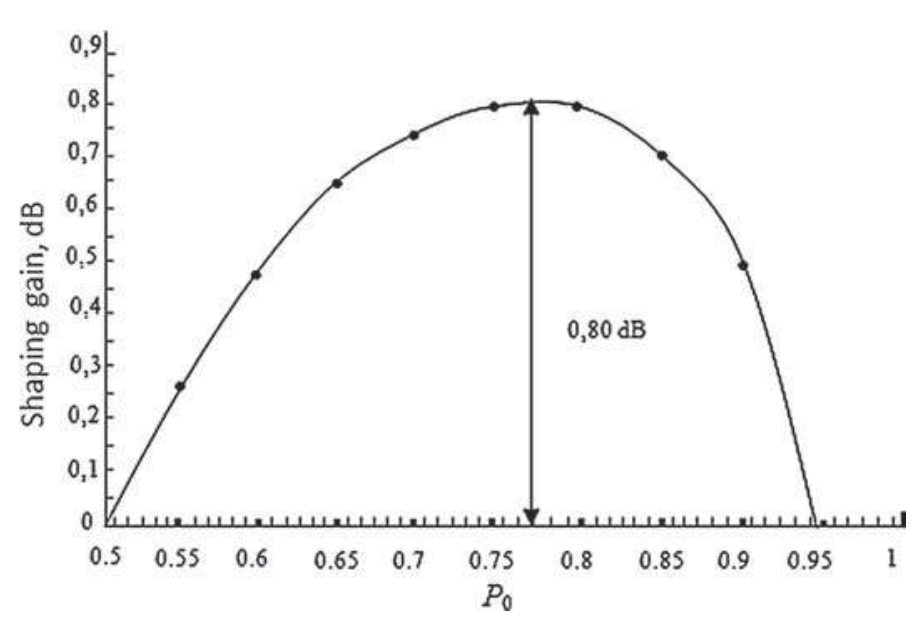

Fig. 4 Variation of the achievable shaping gain against $P_{0}$ for the proposed system, where $I=3$ and $m=4$ (16-PAM)

in Eq. (12), the term $N_{0}$ is computed using:

$$
N_{0}=\frac{4^{m-1}\left(7-6 \cdot P_{0}\right)-1}{3 \cdot \gamma_{s}}
$$

where $\gamma_{s}$ is the average signal to noise ration (SNR) per transmitted signal. Numerical integration of (13) via the Monte Carlo method allows us to determine the achievable shaping gain for any desired mutual information I and any values of parameters $\mathrm{m}$ and $P_{0}$. Figure 4 shows the variation of the shaping gain as a function of $P_{0}$, when $I=3 \mathrm{bit} / \mathrm{dim}$ and $m=4$ (16-PAM). It is seen that the simple partitioning method considered in this paper can offer shaping gain larger than $0.7 \mathrm{~dB}$ provided that the shaping code is designed so that $P_{0}$ ranges from 0.69 to 0.85 . The maximal value of shaping gain equal to $0.80 \mathrm{~dB}$, and is obtained when $P_{0} \approx 0.78$.

\subsection{Simulation Results}

We simulated the error performance of several 3-bit/dim 16-PAM BITCMs based on four configurations, which are $\left(Q=2, R_{c}=6 / 7\right),\left(Q=4, R_{c}=4 / 5\right),\left(Q=6, R_{c}=18 / 23\right)$ and $\left(Q=8, R_{c}=24 / 31\right)$. The rate $-6 / 7,-4 / 5,-18 / 23$ and $-24 / 31$ re obtained by puncturing a rate- $1 / 3$ turbo code built from two parallel-concatenated 16-sate RSC codes with polynomials $(23,35)$. To ensure optimal error performance, only parity bits are punctured. The size of the pseudo-random interleaver separating both RSC codes, i.e. the length $N$ of a frame of information bits, is equal to 3,000 bits. Turbo coding is performed in 10 iterations, and the MAP algorithm is used to decoding of each RSC code. Gray mapping is used such as maximum protection is offered to information bits.

Figure 5 shows graphs of BER versus $E_{\mathrm{b}} / N_{0}$ for a 3-bit/s/Hz 16-PAM BITCM scheme. The BER curves obtained without shaping is displayed for comparison purposes.

It is seen that the shaping technique described in this paper allows for significant error performance improvement for different values $Q$. For instance, at a BER $=10^{-5}$, we achieve, with the length $Q=2$, shaping gain equal to $0.79 \mathrm{~dB}$. To illustrate the effect of the side information in the final results, we have plotted in the Fig. 5 the BER curves obtained with a 
B. S. Bouazza et al.

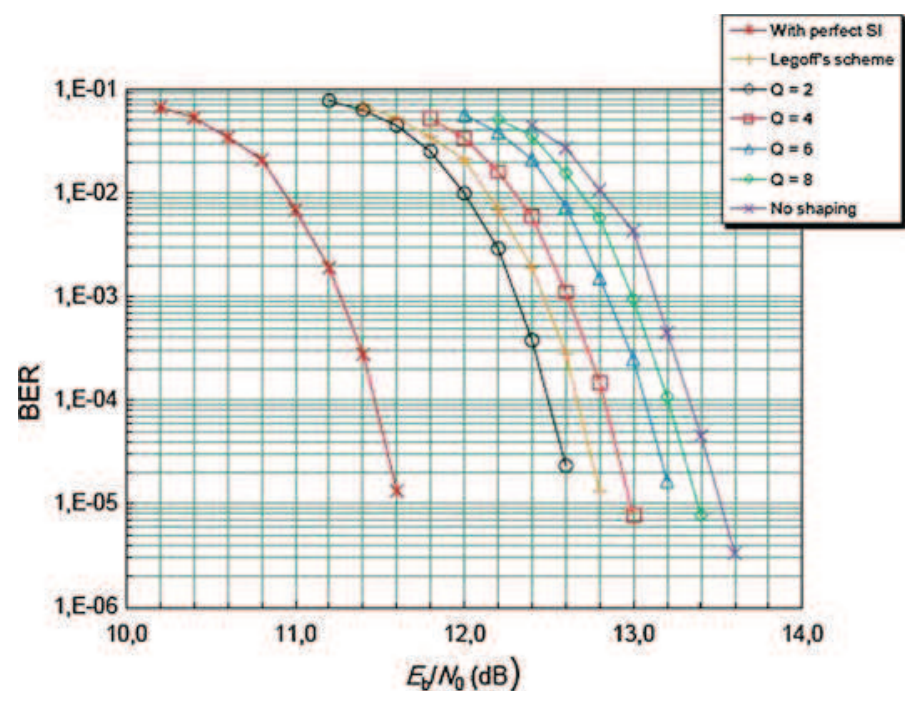

Fig. 5 BER Performance comparisons over AWGN channel between serval 3-bit/dim 16-PAM BITCM schemes

3-bit/dim 16-PAM BITCM system assuming perfect side information and $Q=2$. It is seen that the error rate on the transmitted SI results in a significant error performance degradation of all configurations. For instance, at a BER of $10^{-5}$ and $Q=2$, the performance loss, almost equal to $1.08 \mathrm{~dB}$, which confirm the considerable effect due the side information. We have plotted in Fig. 5 the BER curves obtained with a 3-bit/dim 16-PAM BITCM system designed using Legoff's technique [6]. It is seen that the performance difference between both schemes at a BER $=10^{-5}$ is equal to $0.2 \mathrm{~dB}$. As for the complexity issue, Legoff's scheme is more complex to implement than our system.

\section{Conclusion}

We have presented a very simple technique to combine constellation shaping in the context of BICM. Simulation results show that, in practice, a 3-bit/dim 16-PAM BITCM scheme designed using this technique can achieve shaping gains of approximately $0.79 \mathrm{~dB}$. It is important to mention that our technique preserves most of the simplicity and the flexibility that have made the BICM approach so attractive for many practical applications.

\section{References}

1. Forney, G. D, Jr, Gallager, R. G., Lang, G. R., Longstaff, F. M., \& Qureshi, S. U. (1984). Efficient modulation for band-limited channels. IEEE Journal of Selected Areas in Communication, 2, 632-647.

2. Khandani, A. K., \& Kabaland, P. (1993). Shaping multidimensional signal spaces-Part II: Shell-addressed constellation. IEEE Transactions on Information Theory, 39, 1809-1819.

3. Berrou, C., Glavieux, A., \& Thitimajshima, P. (May 1993). Near Shannon limit error correcting coding and decoding: turbo codes. In Proceedings of the IEEE ICC'93, Geneva, Switzerland, pp. 1064-1070.

4. Sommer, D., \& Fettweis, G. P. (Jan 2000). Signal shaping by non-uniform QAM for AWGN channels and applications using turbo coding. In Proceedings of the ITG conference on source channel coding, pp. 81-86. 


\section{Author's personal copy}

5. Raphaeli, D., \& Gurevitz, A. (2004). Constellation shaping for pragmatic turbo-coded modulation. IEEE Transactions on Communications, 52, 341-345.

6. Le Goff, S. Y., Khoo, B. K., Tsimenidis, C. C., \& Sharif, B. S. (2007). Constellation shaping for bandwidthefficient turbo-coded modulation with iterative receiver. IEEE Transactions on Wireless Communications, 6, 2223-2233.

7. Bouazza, B. S., \& Djebbari, A. (2007). Bit-interleaved coded modulation with iterative decoding using constellation shaping over Rayleigh fading channels. International Journal of Electronics and Communications (AË̈), 61, 405-410.

8. Calderbank, A. R., \& Ozarow, L. H. (1990). Nonequiprobable signaling on the Gaussian channel. IEEE Transactions on Information Theory, 36, 726-740.

9. Caire, G., Taricco, G., \& Biglieri, E. (1998). Bit-interleaved coded modulation. IEEE Transactions on Information Theory, 44, 927-946.

10. LeGoff, S. Y. (2003). Signal constellations for bit-interleaved coded modulation. IEEE Transactions on Information Theory, 49, 307-313.

11. LeGoff, S. Y., Glavieux, A., \& Berrou, C. (May 1994). Turbo codes and high spectral efficiency modulation. In Proceedings of the ICC'94, New Orleans, Louisiana, pp. 645-649

12. Hagenauer, J., Offer, E., \& Papke, L. (1996). Iterative decoding of binary block and convolutional codes. IEEE Transactions on Information Theory, 42, 429-444.

\section{Author Biographies}
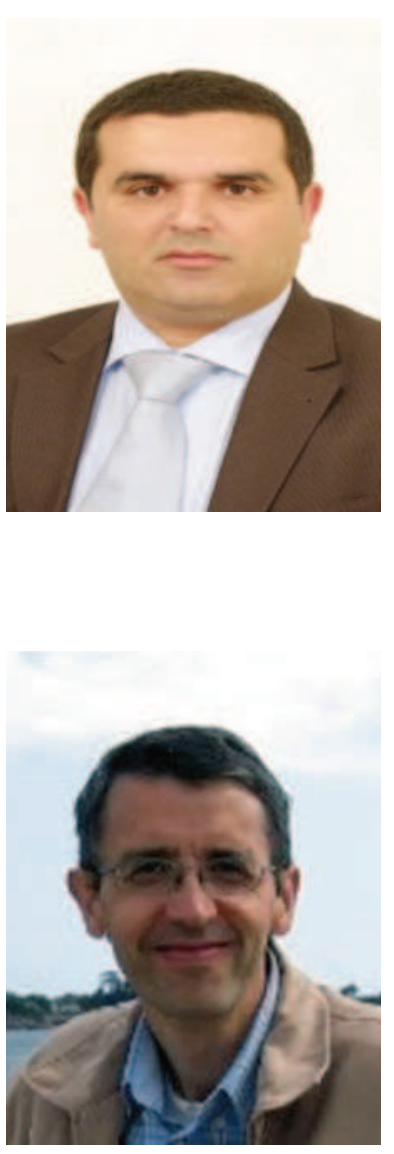

Boubakar S. Bouazza received the B.Sc., M.Sc., and the Ph.D. degrees, all in electrical engineering, from the University of Sidi Bel Abbes, Algeria, in 1996, 1999, and 2006, respectively. Since 1999, he joined the University of Saida (Algeria) first as an assistant professor (1999-2006) and currently as a Lecturer in the Electronics Department. From January 2005 to July 2006, he held visiting position at Ecole Nationale Supérieure de Télécommunications de Bretagne (ENST), Brest, France, as a Ph.D. student. Dr. Bouazza's current research interests are in digital communications, wireless and mobile communications, channel coding, turbo codes and bandwidth-efficient coded modulation schemes.

Stéphane Y. LeGoff received the B.Sc., M.Sc., and Ph.D. degrees, all in electrical engineering, from the University of Western Brittany, Brest, Brittany, France, in 1990, 1991, and 1995, respectively. From 1995 to 1997, he worked as a teacher at the institut Supérieur d'Electronique de Bretagne in Brest . During 1999-2003, Dr. LeGoff was an Assistant Professor at the Emirates Telecommunications Corporation (Etisalat) College of Engineering, UAE. In 2003, he joined the Department of Physics and Electronics at the University of Waikato, Hamilton, New Zealand, as a Senior Lecturer in Electronics. Since March 2005, he has been a Lecturer in the School of Electrical, Electronic, and Computer Engineering at the University of Newcastle upon tyne, UK. Dr. LeGoff also held visiting positions at the Eastern Mediterranean University, Cryprus, during the academic year 1998-1999 and at the Sultan Qaboos University, Oman, from September to December 2004. His research interests include information theory, channel coding, and wireless communications systems. 


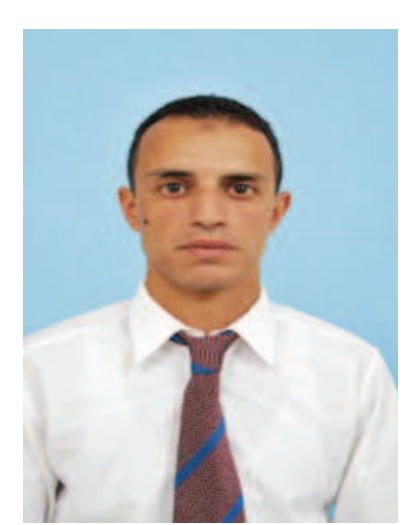

Ahmed Garadi received the B.Sc., and M.Sc., Eng. In Electronic from the University of SAIDA, Algeria in 2006 and University of Sidi Bel Abbes, Algeria, 2010, respectively. Since 2012, he joined the University of Saida (Algeria) first as an assistant professor until today.

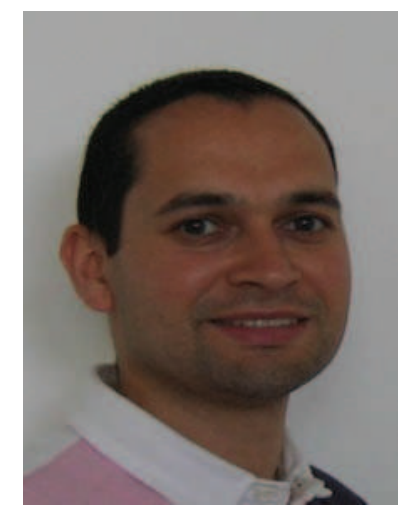

Clency Perrine received his M.S., degree in telecommunications in 2002 and his Ph.D. in telecommunications and signal processing in 2005 from the University of Rennes 1 . His thesis deals with an operational system for images transmission in HF band ( $3\{30 \mathrm{MHz}$ ). In September 2006, he joined the XLIM-SIC laboratory in Poitiers as an associate professor. His research interests are focused on Digital Communication Systems and joint source/channel coding schemes for images transmission through non-stationary wireless channels.

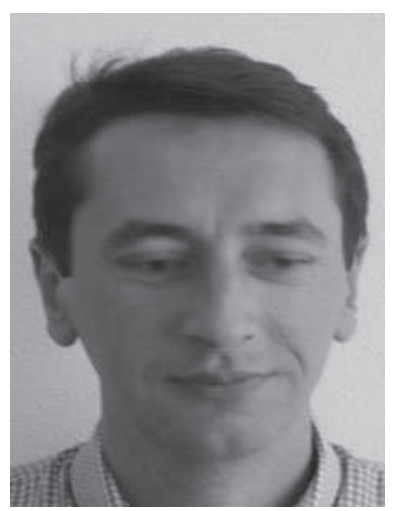

勧 Springer
Rodolphe Vauzelle received the Ph.D. degree in mobile radio communication from the University of Poitiers, in 1994. During ten years, he is professor assistant in the electrical department of the University of Poitiers. Since 2005, he is Professor at the University of Poitiers in the department of Electronics. He develops its research activities in the SIC-XLIM (Signal Image and Communication) laboratory of the University of Poitiers. His research interests focus the realistic simulation of the physical layer of ad'hoc network based on SISO and MIMO channel. 